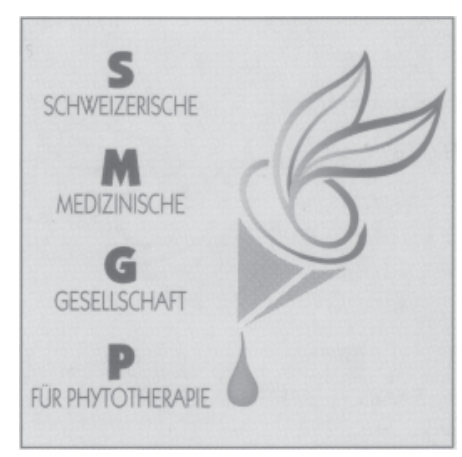

\section{SMGP-Spätsommer-Exkursion ins Maderanertal}

Unter der stets sehr kompetenten Führung von Dr. med. dipl. Natw. Peter Kälin und Prof. Dr. sc. Adrian Leutmann, die vor der eigentlichen Wanderung auch geografische und kulturgeschichtliche Zusammenhänge des Maderanertals erwähnten, wanderten wir verschiedene Standorte an, an denen uns die beiden Experten die botanischen und die phytotherapeutischen Aspekte und Eigenheiten der gefunden Arzneipflanzen erklärten. Dabei wurden auch immer wieder Exkurse in die Volksheilkunde gemacht und zum Beispiel der auf fetten Wiesen wachsende Gute Heinrich (Chenopodium Bonus henricus) vorgestellt, eine von Sennen bei Rindern oft gegen verletzungsbedingte Entzündungen verwendete Pflanze. Am selben Standort wächst der Rot-

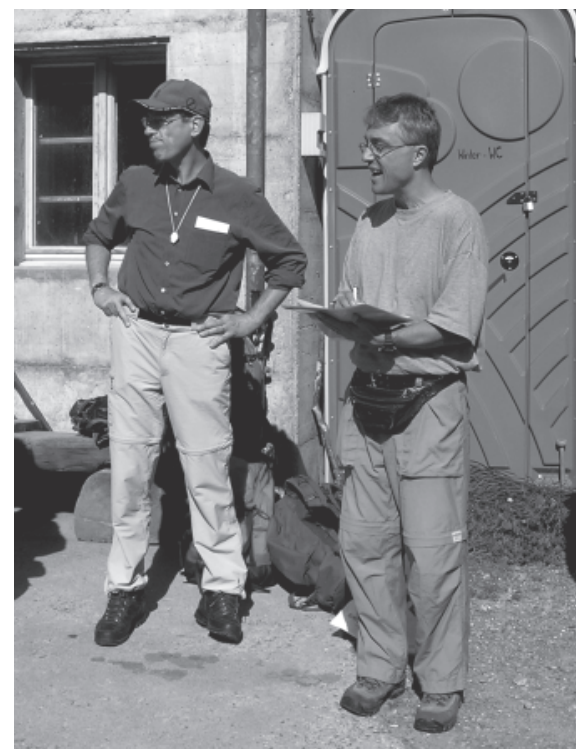

Abb. 1. Die beiden Exkursionsleiter erklären den Ablauf des Tages.

\section{Gesellschaftsmitteilungen · Society Bulletins}

Forsch Komplementärmed 2007;14:319-320 klee, Trifolium pratense, über dessen Eignung bei menopausalen Beschwerden eine Diskussion entstand. Bei Euphrasia roskoviana meldete sich Markus Schreier, «SMGP-Augenarzt» zu Worte und erläuterte die Diskrepanz zwischen dem deutschen und lateinischen Namen der Pflanze. Weiter erklärte er, dass in der Augenheilkunde neben dieser Euphrasia-Art auch E. officinalis und E. minor verwendet werden. Am Golzernsee wurden für den Standort typische Pflanzen vorgestellt. Anschließend wanderten wir noch $\mathrm{zu}$ einem etwas höher gelegenen Moorhang, wo Adrian Leutmann den Unterschied zwischen einem Hoch- und einem Flachmoor und die extremen Lebensbedingungen von Hochmoorpflanzen erklärte. Nur wenige Pflanzen können sich in dieser extrem sauren Umgebung ansiedeln, so etwa der als Arzneipflanze bekannte Sonnentau, Drosera

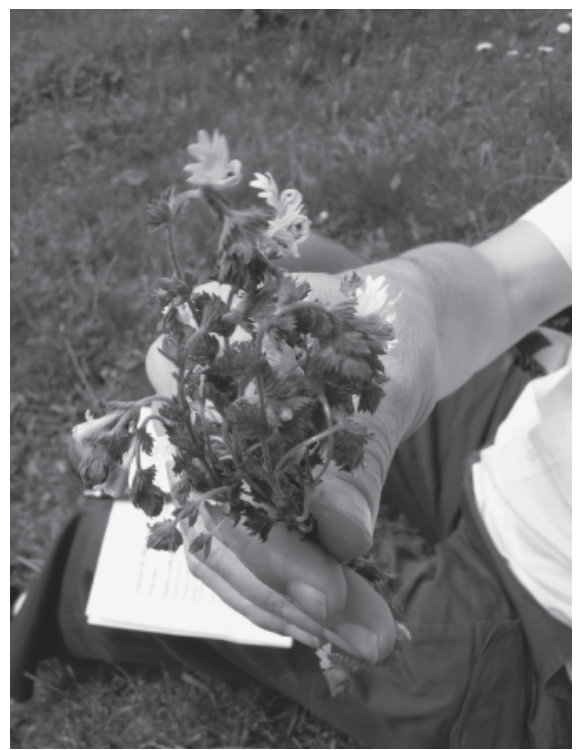

Abb. 2. Euphrasia roskoviana. rotundifolia oder das Pfeifengras, Molinia coerulea. In diesem Moorgebiet fanden wir auch den Eisenhut, Aconitus napellum, die giftigste einheimische Pflanze. Auch hier gab es eine kulturhistorische Diskussion. Es wurde über die Wirkungsweise von Schierlingsamen, Conium maculatum, gesprochen, mit dem Sokrates umgebracht wurde.

Wer nicht dabei war hat einen wunderschönen Tag verpasst - anerkannte Fortbildung im Freien, wer kann das schon bieten? Vielleicht sind die Exkursionen aber im nächsten Jahr wieder einmal ausgebucht.

Christoph Bachmann

Luzern

Korrespondenzadresse:

Geschäftsstelle SMGP

Prof. Dr. sc. nat. Beat Meier

Zürcher Hochschule für angewandte

Wissenschaften

8820 Wädenswil

E-mail beat.meier@zhaw.ch; www.smgp.ch

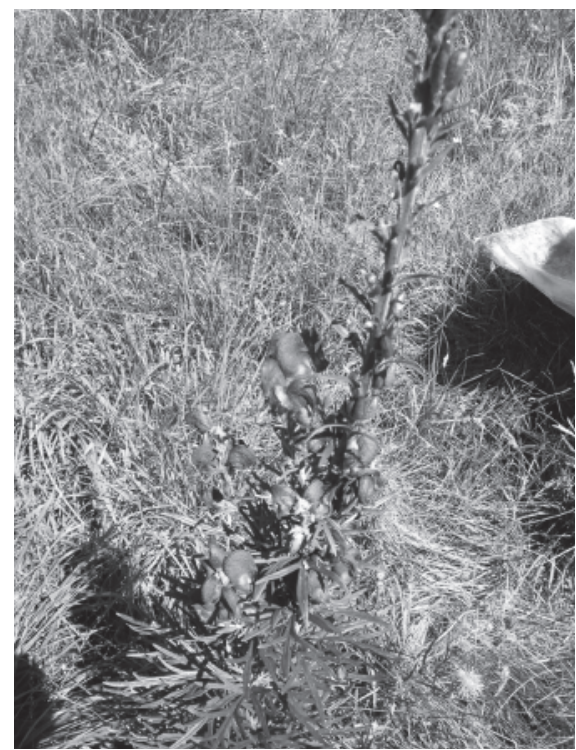

Abb. 3. Aconitum napellus, Eisenhut, die giftigste einheimische Pflanze. 


\section{Excursion pharmaco-botanique, le 9 juin 2007 à Leysin}

L'excursion pharmaco-botanique du 9 pants ont vécu une journée mémorable juin était vouée à la Fondation Gentiana à Leysin ainsi qu'au lac d'Aï. Les partici-
Prof. Kurt Hostettmann et le Dr. Marston de l'université de Genève.
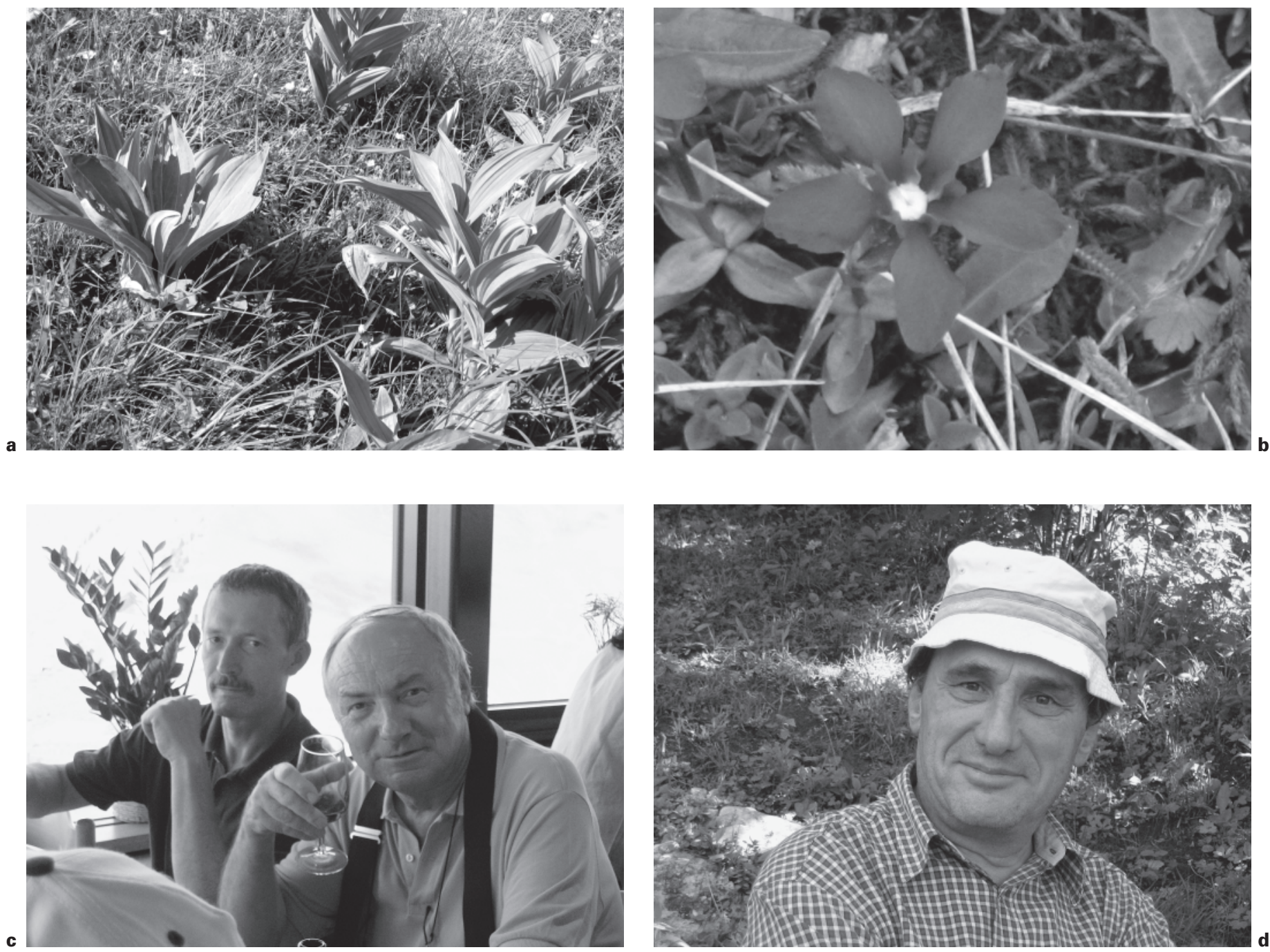

Abb. 5. a Gentiana lutea et veratrum album poussent l'un à côté de l'autre et peuvent donc être confondus. Gentiana lutea und Veratrum album wachsen nebeneinander und können verwechselt werden. b Gentiana kochiana au-dessus de Leysin (1900 m). Gentiana kochiana oberhalb von Leysin $(1900$ m) c Nos guides/unsere Führer: Prof. K. Hostettmann et Dr. A. Marston, Université de Genève. d Dr. Damien Decrey, membre du comité. 